\title{
Haldane Phases and Ferrimagnetic Phases with Spontaneous Translational Symmetry Breakdown in Distorted Mixed Diamond Chains with Spins 1 and $1 / 2$
}

\author{
Kazuo Hida*, Ken’ichi TAKano ${ }^{1}$, and Hidenori Suzuki ${ }^{1 \dagger}$ \\ Division of Material Science, Graduate School of Science and Engineering, \\ Saitama University, Saitama, Saitama, 338-8570 \\ ${ }^{1}$ Toyota Technological Institute, Tenpaku-ku, Nagoya 468-851
}

(Received February 28, 2018)

\begin{abstract}
The ground states of two types of distorted mixed diamond chains with spins 1 and $1 / 2$ are investigated using exact diagonalization, DMRG, and mapping onto low-energy effective models. In the undistorted case, the ground state consists of an array of independent spin1 clusters separated by singlet dimers. The lattice distortion induces an effective interaction between cluster spins. When this effective interaction is antiferromagnetic, several Haldane phases appear with or without spontaneous translational symmetry breakdown (STSB). The transition between the Haldane phase without STSB and that with $(n+1)$-fold STSB $(n=1$, 2 , and 3) belongs to the same universality class as the $(n+1)$-clock model. In contrast, when the effective interaction is ferromagnetic, the quantized and partial ferrimagnetic phases appear with or without STSB. An effective low-energy theory for the partial ferrimagnetic phase is presented.
\end{abstract}

KEYWORDS: mixed diamond chain, distortion, frustration, Haldane phase, spontaneous translational symmetry breakdown, partial ferrimagnetism

\section{Introduction}

Quantum magnetism in frustrated spin systems is a rapidly developing field of condensed matter physics. ${ }^{1,2)}$ At first glance, one would expect that geometrical frustration enhances quantum fluctuation and drives an ordered state into a disordered state. However, recent progress in this field of physics has shown that this simple intuition is not always valid and that geometrical frustration induces a variety of exotic quantum phenomena, which are not easily predicted. Under an appropriate condition, it even stabilizes an unexpected magnetic long range order such as the frustration-induced ferrimagnetic and spin nematic orders.

To understand magnetism under the interplay of geometrical frustration and quantum fluctuation, it is desirable to begin with typical spin models with exact solutions. Among them, there exist a class of models whose ground states are exactly written down as spin cluster solid (SCS) states because of frustration. A SCS state is a tensor product state of exact local eigenstates of cluster spins. Well-known examples are the Majumdar-Ghosh model $^{3)}$ whose ground state is a prototype of spontaneously dimerized phases in one-dimensional frustrated magnets $^{4)}$ and the Shastry-Sutherland model ${ }^{5)}$ which corresponds to the material $\mathrm{SrCu}_{2}\left(\mathrm{BO}_{3}\right)_{2} \cdot{ }^{6,7)}$ In these models, the spin clusters are singlet dimers.

The diamond chain is another frustrated spin chain with exact SCS ground states. The lattice structure is shown in Fig. 1. In a unit cell, there are two kinds of nonequivalent lattice sites occupied by spins with magnitudes $S$ and $\tau$; we denote the set of magnitudes by $(S$, $\tau)$. One of the authors and coworkers ${ }^{8,9)}$ introduced this

*E-mail address: hida@phy.saitama-u.ac.jp

$\dagger$ Present address: Department of Physics, College of Humanities and Sciences, Nihon University, Setagaya-ku, Tokyo 156-8550

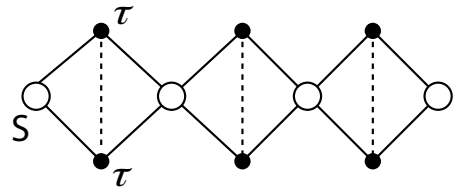

Fig. 1. Structure of the diamond chain. Spin magnitudes in a unit cell are indicated by $S$ and $\tau$; we denote the set of magnitudes by $(S, \tau)$. The PDC is the case of $S=\tau$, while the MDC is the case of $S=2 \tau$ with an integer or half-odd integer $\tau$.

lattice structure and generally investigated the case of $(S, S)$, i.e., the pure diamond chain (PDC). Any PDC is shown to have at least one exact SCS ground-state phase where each spin cluster has spin 0. Particularly, in the case of $(1 / 2,1 / 2)$, they determined the full phase diagram of the ground state by combining rigorous arguments with numerical calculations. After that, Niggemann et al. ${ }^{10,11)}$ argued about a series of diamond chains with $(S, 1 / 2)$. As for the special case of $(1 / 2,1 / 2)$, they reproduced the results of ref. 9 .

The mixed diamond chain (MDC) is defined as a diamond chain with $(S, S / 2)$ for the integer $S .{ }^{12)}$ The special case of $(1,1 / 2)$ was first investigated by Niggemann et al. ${ }^{10,11)}$ They considered it as one of the series of models with $(S, 1 / 2)$. Recently, extensive investigation on the MDC has been carried out by the present authors. ${ }^{12-14)}$ The MDC is of special interest among diamond chains, because only the MDC has the Haldane phase in the absence of frustration, so that we can observe the transition from the Haldane phase to a SCS phase induced by frustration. In contrast, diamond chains of other types have ferrimagnetic ground states for weak frustration.

The features common to all types of diamond chains are their infinite number of local conservation laws and 
more than two different types of exact SCS ground states that are realized depending on the strength of frustration. For example, $S=1 / 2 \mathrm{PDC}$ has a nonmagnetic phase accompanied by spontaneous translational symmetry breakdown (STSB) and a paramagnetic phase without STSB. This model also has a ferrimagnetic ground state in the less frustrated region. ${ }^{9}$ ) On the other hand, the MDC with spins 1 and $1 / 2$ has 3 different paramagnetic phases accompanied by STSB and one paramagnetic phase without STSB. This model also has a nonmagnetic Haldane ground state in a less frustrated region. ${ }^{10,12)}$ The SCS structures of the ground states are also reflected in characteristic thermal properties, as reported in ref. 13 .

Modifications of the PDC and MDC have been examined by many authors. Among them, the spin 1/2 PDC with distortion has been thoroughly investigated by numerical methods. ${ }^{15-17)}$ It is found that azurite, a natural mineral, consists of distorted PDCs with spin $1 / 2$ and that the magnetic properties of this material have been experimentally studied in detail. ${ }^{18,19)}$ Other materials have also been reported. ${ }^{20,21)}$ The diamond chain is one of the simplest models compatible with the 4-spin cyclic interaction. The effects of this type of interaction on PDC have recently been investigated by Ivanov et al. ${ }^{22}$ ) The present authors also investigated the MDC with bond-alternating distortion and found an infinite series of ground states with STSB. ${ }^{14)}$ In addition, as reviewed in ref. 14, the MDC is related to other important models of frustrated magnetism such as the dimerplaquette model, ${ }^{23-28)}$ frustrated Heisenberg ladders, ${ }^{29)}$ hybrid diamond chains consisting of Heisenberg bonds and Ising bonds, ${ }^{30,31)}$ and an Ising model on a hierarchical diamond lattice. ${ }^{32)}$ Among them, the dimer-plaquette chain with ferromagnetic interplaquette interaction reduces to the MDC in the limit of strong interplaquette interaction. ${ }^{28)}$

Thus far, in spite of the theoretical relevance of the MDC, no materials described by the MDC have been found. Nevertheless, synthesizing MDC materials is not an unrealistic expectation in view of the success of the synthesis of many low dimensional bimetallic magnetic compounds $^{33)}$ and organic magnetic compounds. ${ }^{34)}$ In general, it is natural to expect that the lattice is possibly distorted in real MDC compounds as in azurite. From this viewpoint, it is important to present theoretical predictions on the ground state of distorted MDCs to widen the range of candidate materials of MDC and to raise the possibility of their synthesis.

We begin by classifying the distortion patterns by the normal modes of each diamond unit. Excluding two translations and one rigid body rotation, we have 5 normal modes as depicted in Fig. 2 within the diamond plane. A distorted MDC may be realized as a result of the collective softening of these normal modes. In particular, the distortion patterns in (a) and (b) break the local conservation laws that hold in the undistorted MDC. Hence, these distortions induce effective interactions between the cluster spins in the whole lattice, and may form novel exotic phases. We investigate these interesting cases in the present paper. In what follows, we name

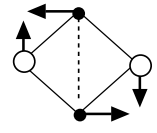

(a)
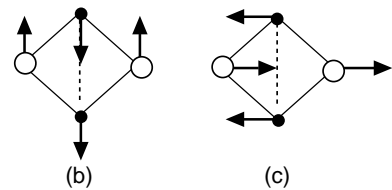

(c)

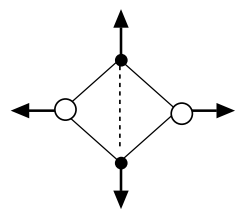

(d)

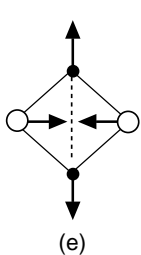

Fig. 2. Displacement modes of a diamond unit.

(a)

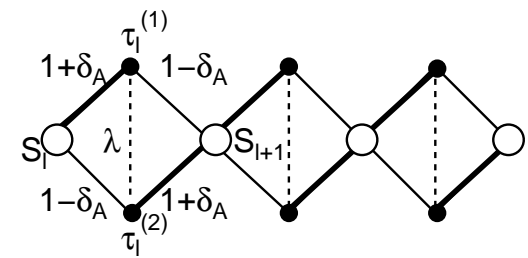

(b)

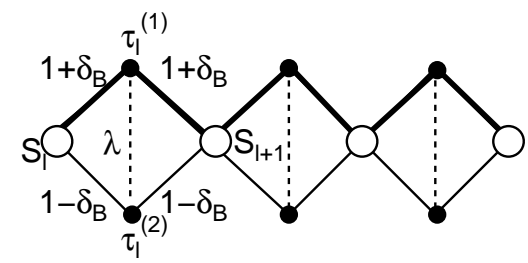

Fig. 3. Structures of MDC with $S=1$ and $\tau^{(1)}=\tau^{(2)}=1 / 2$ with (a) type A and (b) type B distortions.

the distortion patterns in (a) and (b) as type A and type $\mathrm{B}$, respectively. The MDCs with type A and type B distortions are depicted in Figs. 3(a) and 3(b), respectively. The distortion patterns in Figs. 2(d) and 2(e) do not change the geometry of the original undistorted MDC. The distortion pattern in Fig. 2(c) is of another interest, since it induces the bond alternation in the undistorted MDC without breaking the local conservation laws. This case has been investigated separately and published in a previous paper. ${ }^{14)}$

This paper is organized as follows. In $\S 2$, the Hamiltonians for the MDCs with the type A and type B distortions are presented, and the structure of the ground states of the MDC without distortion is summarized. The ground-state phases for the MDC with the type A distortion are discussed in $\S 3$, and those for the MDC with the type $\mathrm{B}$ distortion are discussed in $\S 4$. The last section is devoted to summary and discussion.

\section{Hamiltonian}

The MDCs with the type A and type B distortions are described, respectively, by the following Hamiltonians:

$$
\begin{aligned}
\mathcal{H}_{\mathrm{A}}= & \sum_{l=1}^{N}\left[\left(1+\delta_{\mathrm{A}}\right) \boldsymbol{S}_{l} \boldsymbol{\tau}_{l}^{(1)}+\left(1-\delta_{\mathrm{A}}\right) \boldsymbol{\tau}_{l}^{(1)} \boldsymbol{S}_{l+1}\right. \\
& \left.+\left(1-\delta_{\mathrm{A}}\right) \boldsymbol{S}_{l} \boldsymbol{\tau}_{l}^{(2)}+\left(1+\delta_{\mathrm{A}}\right) \boldsymbol{\tau}_{l}^{(2)} \boldsymbol{S}_{l+1}+\lambda \boldsymbol{\tau}_{l}^{(1)} \boldsymbol{\tau}_{l}^{(2)}\right],
\end{aligned}
$$




$$
\begin{aligned}
\mathcal{H}_{\mathrm{B}}= & \sum_{l=1}^{N}\left[\left(1+\delta_{\mathrm{B}}\right) \boldsymbol{S}_{l} \boldsymbol{\tau}_{l}^{(1)}+\left(1+\delta_{\mathrm{B}}\right) \boldsymbol{\tau}_{l}^{(1)} \boldsymbol{S}_{l+1}\right. \\
& \left.+\left(1-\delta_{\mathrm{B}}\right) \boldsymbol{S}_{l} \boldsymbol{\tau}_{l}^{(2)}+\left(1-\delta_{\mathrm{B}}\right) \boldsymbol{\tau}_{l}^{(2)} \boldsymbol{S}_{l+1}+\lambda \boldsymbol{\tau}_{l}^{(1)} \boldsymbol{\tau}_{l}^{(2)}\right],
\end{aligned}
$$

where $\boldsymbol{S}_{l}$ is the spin-1 operator, and $\boldsymbol{\tau}_{l}^{(1)}$ and $\boldsymbol{\tau}_{l}^{(2)}$ are the spin- $1 / 2$ operators in the $l$ th unit cell. The parameter $\delta_{\mathrm{A}}\left(\delta_{\mathrm{B}}\right)$ represents the strength of type A (type B) distortion, and is taken to be nonnegative without spoiling generality. The number of unit cells is denoted by $N$, and then the total number of sites is $3 N$. We will consider these systems in the large $N$ limit.

For $\delta_{\mathrm{A}}=0$ and $\delta_{\mathrm{B}}=0$, both eqs. (1) and (2) reduce to the undistorted MDC Hamiltonian,

$$
\mathcal{H}_{0}=\sum_{l=1}^{N}\left[\boldsymbol{S}_{l} \boldsymbol{T}_{l}+\boldsymbol{T}_{l} \boldsymbol{S}_{l+1}+\frac{\lambda}{2}\left(\boldsymbol{T}_{l}^{2}-\frac{3}{2}\right)\right]
$$

with the composite spin operators $\boldsymbol{T}_{l} \equiv \boldsymbol{\tau}_{l}^{(1)}+\boldsymbol{\tau}_{l}^{(2)}$. Before going into the analysis of the distorted MDC, we briefly summarize the ground-state properties of the Hamiltonian (3) reported in ref. 12 for convenience.

(i) $\boldsymbol{T}_{l}^{2}$ commutes with the Hamiltonian $\mathcal{H}_{0}$ for any $l$. Therefore, the composite spin magnitude $T_{l}$ defined by $\boldsymbol{T}_{l}^{2}=T_{l}\left(T_{l}+1\right)$ is a good quantum number that takes the values 0 or 1 . Hence, each energy eigenstate has a definite set of $\left\{T_{l}\right\}$, i.e. a sequence of 0 's and 1's with length $N$. A pair of $\boldsymbol{\tau}_{l}^{(1)}$ and $\boldsymbol{\tau}_{l}^{(2)}$ with $T_{l}=0$ is called a dimer. A cluster including $n$ successive $T_{l}=1$ pairs bounded by two $T_{l}=0$ pairs is called a cluster- $n$. The cluster- $n$ is equivalent to an antiferromagnetic spin-1 Heisenberg chain of length $2 n+1$ with open boundary condition. Since a cluster- $n$ is decomposed into a sublattice consisting of $n+1$ sites with $\boldsymbol{S}_{l}$ 's and that consisting of $n$ sites with $\boldsymbol{T}_{l}$ 's, the ground states of a cluster- $n$ are spin triplet states with total spin unity on the basis of the Lieb-Mattis theorem. ${ }^{35,36)}$ This implies that each cluster- $n$ carries a spin-1 in its ground state.

(ii) There appear 5 distinct ground-state phases called dimer-cluster- $n$ (DC $n$ ) phases with $n=0,1,2,3$, and $\infty$. The $\mathrm{DC} n$ state is an alternating array of dimers and cluster- $n$ 's. The phase boundary $\lambda_{\mathrm{c}}\left(n, n^{\prime}\right)$ between $\mathrm{DC} n$ and $\mathrm{DC} n^{\prime}$ phases are

$$
\begin{aligned}
& \lambda_{c}(0,1)=3 \\
& \lambda_{c}(1,2) \simeq 2.660425045542, \\
& \lambda_{c}(2,3) \simeq 2.58274585704, \\
& \lambda_{c}(3, \infty) \simeq 2.5773403291,
\end{aligned}
$$

where $\lambda_{c}(0,1)$ is obtained analytically and other values are calculated numerically.

(iii) In the $\mathrm{DC} \infty$ ground state realized for $\lambda<\lambda_{\mathrm{c}}(3, \infty)$, $T_{l}=1$ for all $l$. This state is not accompanied by STSB and is equivalent to the Haldane state of an antiferromagnetic spin-1 Heisenberg chain with infinite length.

(iv) Each of the DC $n$ states with $0 \leq n \leq 3$ realized for $\lambda>\lambda_{\mathrm{c}}(3, \infty)$ is a uniform array of cluster- $n$ 's with a common value of $n$ and dimers in between. In the DC $n$ phase with $1 \leq n \leq 3,(n+1)$-fold STSB takes place. In the DC0 phase, no translational symmetry is broken.

In what follows, we numerically examine various aspects of the type A and type B distortion effects on the MDC. Because the DC3 phase is only realized within a very narrow interval of $\lambda$, it is difficult to analyze the effect of distortion numerically in this phase. Hence, we do not consider the DC3 phase in the following numerical analysis.

\section{Ground-State Properties of the MDC with Type A Distortion}

\subsection{Weak distortion regime}

We now inspect the nature of the effective interaction between two cluster- $n$ 's separated by a dimer consisting of $\boldsymbol{\tau}_{l}^{(1)}$ and $\boldsymbol{\tau}_{l}^{(2)}$ in the presence of the weak type A distortion. For $\delta_{\mathrm{A}}>0, \boldsymbol{S}_{l}\left(\boldsymbol{S}_{l+1}\right)$ tends to be antiparallel to $\boldsymbol{\tau}_{l}^{(1)}\left(\boldsymbol{\tau}_{l}^{(2)}\right)$ rather than to $\boldsymbol{\tau}_{l}^{(2)}\left(\boldsymbol{\tau}_{l}^{(1)}\right)$, as is known from Fig. 3(a). The spins $\boldsymbol{\tau}_{l}^{(1)}$ and $\boldsymbol{\tau}_{l}^{(2)}$ are antiparallel to each other because they form a singlet dimer. Therefore, $\boldsymbol{S}_{l}$ and $\boldsymbol{S}_{l+1}$ tend to be antiparallel to each other. In each cluster- $n$, the number of spins $\boldsymbol{S}_{l}$ 's is larger than the number of composite spins $\boldsymbol{T}_{l}$ 's by one. Hence, from the Lieb-Mattis theorem, ${ }^{35)}$ the total spin of the ground state of the cluster- $n$ points to the same direction as the $\boldsymbol{S}_{l}$ 's belonging to that cluster- $n$. Therefore, the total spins of the cluster- $n$ 's on both sides of the dimer also tend to be antiparallel to each other. Thus, the effective coupling between the spins of neighboring cluster- $n$ 's is antiferromagnetic. This physical argument will be numerically ensured below.

In general, the interaction between two spins with a magnitude of 1 is the sum of bilinear and biquadratic terms. Therefore, the effective Hamiltonian for cluster$n$ 's in the phase that continues to the $\mathrm{DC} n$ phase in the limit of $\delta_{\mathrm{A}} \rightarrow 0$ is written as

$$
\begin{aligned}
\mathcal{H}^{\mathrm{eff}} & =\sum_{i=1}^{N_{\mathrm{c}}} \mathcal{H}^{\mathrm{eff}}(i, i+1), \\
\mathcal{H}^{\mathrm{eff}}(i, i+1) & =J_{\mathrm{eff}} \hat{\boldsymbol{S}}_{i} \hat{\boldsymbol{S}}_{i+1}+K_{\mathrm{eff}}\left(\hat{\boldsymbol{S}}_{i} \hat{\boldsymbol{S}}_{i+1}\right)^{2},
\end{aligned}
$$

where $\hat{\boldsymbol{S}}_{i}$ is the total spin of the $i$-th cluster- $n$ with a magnitude of $1, N_{\mathrm{c}}$ is the total number of cluster-n's, and $J_{\text {eff }}$ and $K_{\text {eff }}$ are effective coupling constants. From symmetry consideration, the signs of $\delta_{\mathrm{A}}$ does not affect the sign of the effective coupling constants. Hence, these coupling constants are of the order of $\delta_{\mathrm{A}}^{2}$ for small $\delta_{\mathrm{A}}$. We numerically calculated the ground-state energy of a pair of cluster- $n$ 's with total spin $S_{\text {tot }}$, and compared it with the corresponding eigenvalues of $\mathcal{H}^{\text {eff }}(i, i+1)$. Then we confirmed that $J_{\text {eff }} / \delta_{\mathrm{A}}^{2}$ and $K_{\text {eff }} / \delta_{\mathrm{A}}^{2}$ are almost independent of $\delta_{\mathrm{A}}$ typically for $\delta_{\mathrm{A}}<0.002$. The constant values of $J_{\text {eff }} / \delta_{\mathrm{A}}^{2}$ and $K_{\mathrm{eff}} / \delta_{\mathrm{A}}^{2}$ are shown in Fig. 4 for three phases $(n=0,1$, and 2), which will be explained below.

Because the effective coupling constants satisfy $0<$ $K_{\text {eff }} / J_{\text {eff }}<1$, the ground state is the Haldane state for 

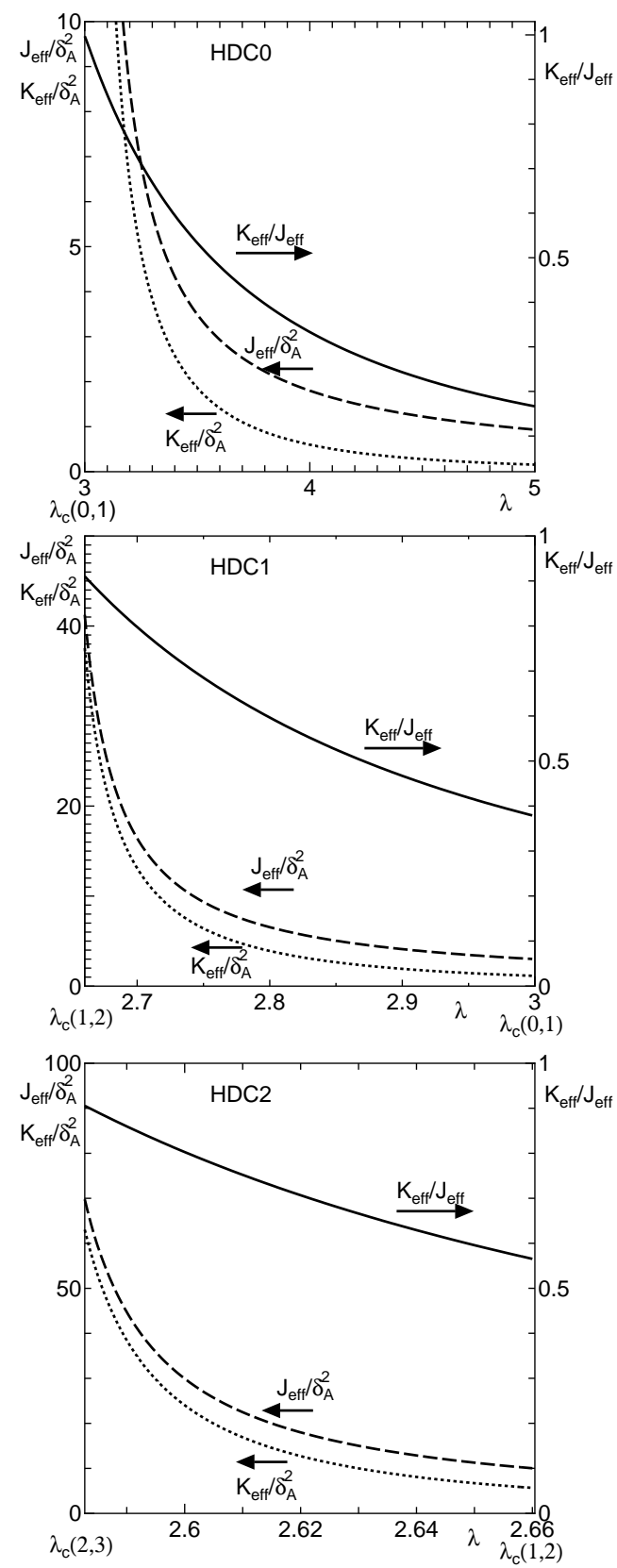

Fig. 4. Effective bilinear interaction $\left(J_{\text {eff }}\right)$ and biquadratic interaction $\left(K_{\text {eff }}\right)$ between spin clusters for small $\delta_{A}$ in HDC0, HDC1, and HDC2 phases from top to bottom. The ratio $K_{\text {eff }} / J_{\text {eff }}$ is also shown.

small $\delta_{\mathrm{A}} .{ }^{37)}$ In the Haldane state, each spin-1 degree of freedom is carried by a cluster- $n$ rather than by a single spin. We call the state the Haldane DCn (HDCn) state. In the HDC $n$ state with $n \geq 1$, the $(n+1)$-fold translational symmetry is spontaneously broken unlike the conventional Haldane state without STSB. Both the HDC0 state for $\lambda>\lambda_{\mathrm{c}}(0,1)$ and the HDC $\infty$ state for $\lambda<\lambda_{\mathrm{c}}(3, \infty)$ are the Haldane states without STSB. In particular, the HDC $\infty$ state continues from the Haldane state $(\mathrm{DC} \infty$ state) of the undistorted MDC mentioned in $\S 2 .{ }^{12)}$ (a)

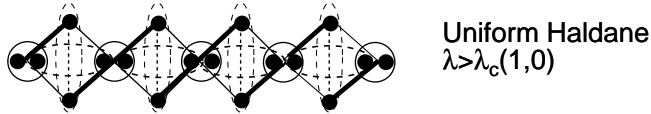

(b)

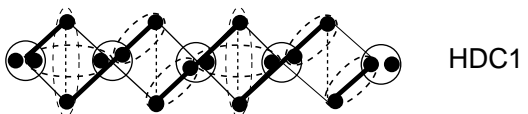

(c)

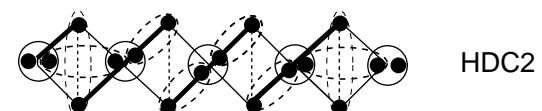

(d)

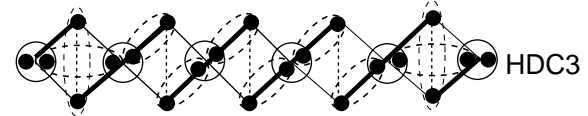

(e)

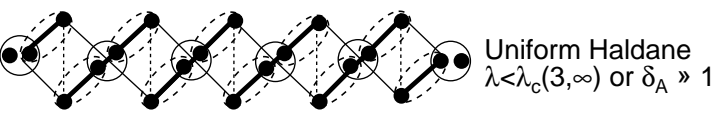

Fig. 5. Valence bond structures of the ground states of all phases for the MDC with type A distortion. A small filled circle represents a spin with a magnitude of $1 / 2$. An original spin with a magnitude of 1 is represented by two decomposed $1 / 2$ spins in an open circle indicating the symmetrization. A valence bond is represented by a dashed oval.

\subsection{Connection to the strong distortion regime}

In the strong distortion regime of $\delta_{\mathrm{A}} \rightarrow 1$ and small $\lambda$, the three spins $\boldsymbol{\tau}_{l-1}^{(2)}, \boldsymbol{S}_{l}$, and $\boldsymbol{\tau}_{l}^{(1)}$ form a singlet cluster. Hence, the ground state is a state with spin gap and without STSB. This nature is common to the HDC0 and $\mathrm{HDC} \infty$ phases in $\S 3.1$. Furthermore, the $\mathrm{HDC} \infty$ state is transformed into the HDC0 state only by rearranging two valence bonds within each diamond unit, as shown in Figs. 5(a) and 5(e). Therefore, the strong distortion, $\mathrm{HDC} 0$, and $\mathrm{HDC} \infty$ regimes are considered to be different parts of a single phase. The continuity of the three regimes will be confirmed by the numerical analysis discussed in $§ 3.3$. In what follows, we call this phase the uniform Haldane (UH) phase as a whole.

\subsection{Numerical phase diagram}

Under the periodic boundary condition, even in the parameter region where STSB takes place, the ground state of a finite chain is a superposition of the symmetrybroken states, and the translational symmetry is recovered. Under the open boundary condition, however, one of the symmetry broken states is selected by the boundary effect. Therefore, we employ the DMRG calculation with the open boundary condition to determine the phase diagram for finite $\delta_{\mathrm{A}}$. The DMRG calculation is carried out using the finite-size algorithm up to 288 sites keeping 200 states in subsystems. We calculate the ground-state expectation values $\left\langle\boldsymbol{T}_{l}^{2}\right\rangle$ and define the effective spin magnitude $T_{l}$ on the $l$-th diagonal bond by $T_{l}\left(T_{l}+1\right)=\left\langle\boldsymbol{T}_{l}^{2}\right\rangle$. A typical $l$ dependence of $T_{l}$ is shown in Fig. 6 in each phase. With the increase in $\delta_{\mathrm{A}}$, the translational symmetry is recovered as expected. For finite $\delta_{\mathrm{A}}$, the ground-state phase is identified from the periodicity in the oscillation of $T_{l}$. In the HDC $n$ phase, the values of 


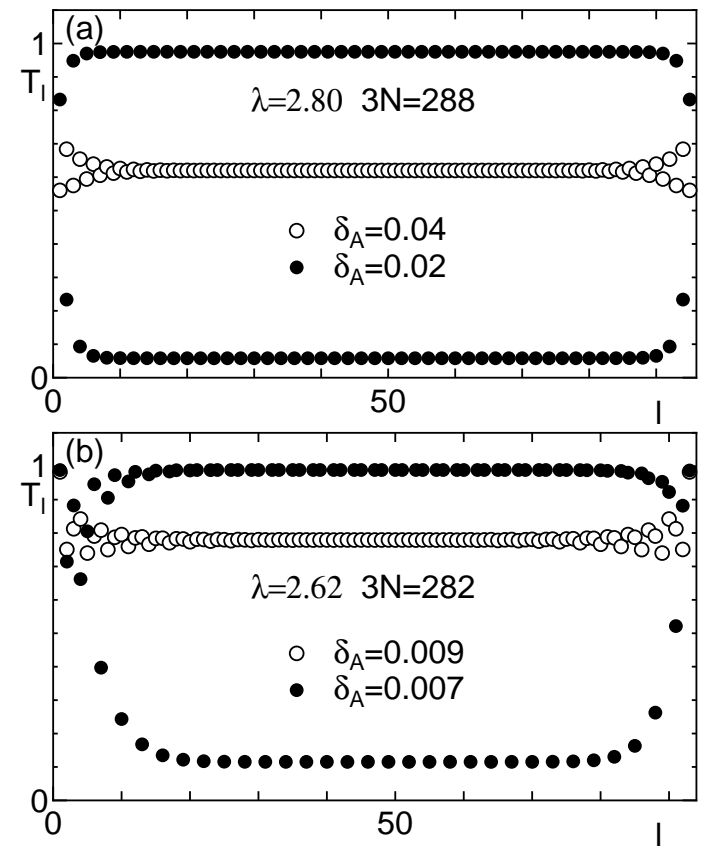

Fig. 6. Profiles of $T_{l}$ for (a) $\lambda=2.8$ and (b) $\lambda=2.62$.
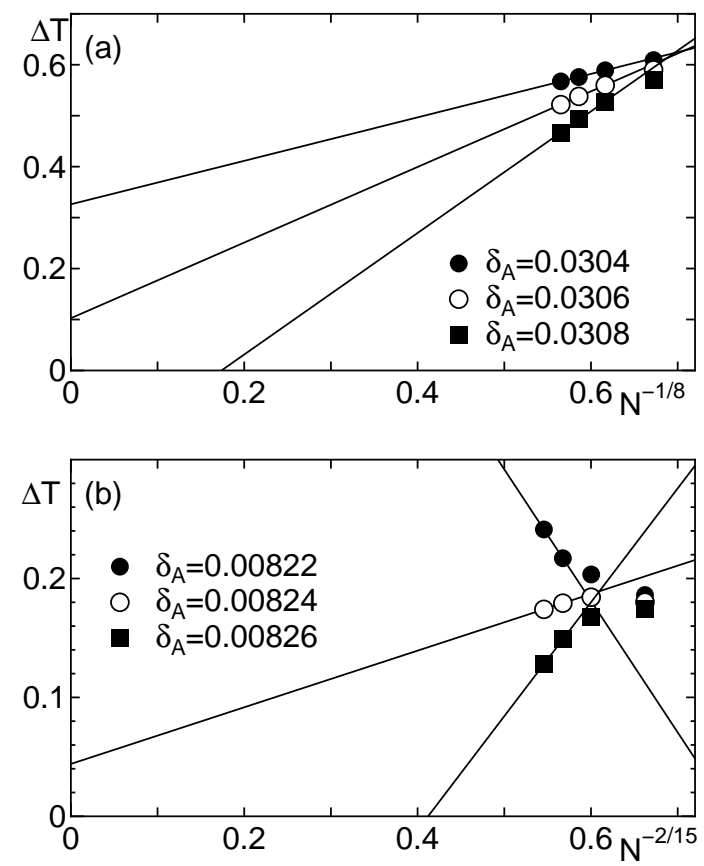

Fig. 7. System size dependences of $\Delta T$ at (a) $\lambda=2.85$ and (b) $\lambda=2.62$. The data are plotted against $N^{-\beta / \nu}$ where $\beta$ and $\nu$ are the critical exponents of the order parameter and correlation length, respectively, for the 2-dimensional (a) Ising and (b) 3clock model.

$T_{l}$ follow the sequence

$$
\cdots T_{\mathrm{S}} \underbrace{T_{\mathrm{L}} \cdots T_{\mathrm{L}}}_{n} T_{\mathrm{S}}, \underbrace{T_{\mathrm{L}} \cdots T_{\mathrm{L}}}_{n} \ldots,\left(T_{\mathrm{L}}>T_{\mathrm{S}}\right) .
$$

Thus, we define the order parameter of the HDC $n$ phase by $\Delta T=T_{\mathrm{L}}-T_{\mathrm{S}}$. In DMRG, $\Delta T$ is measured at the sites closest to the center of the chain.

The valence bond structures for the $\mathrm{HDC} n$ phases as

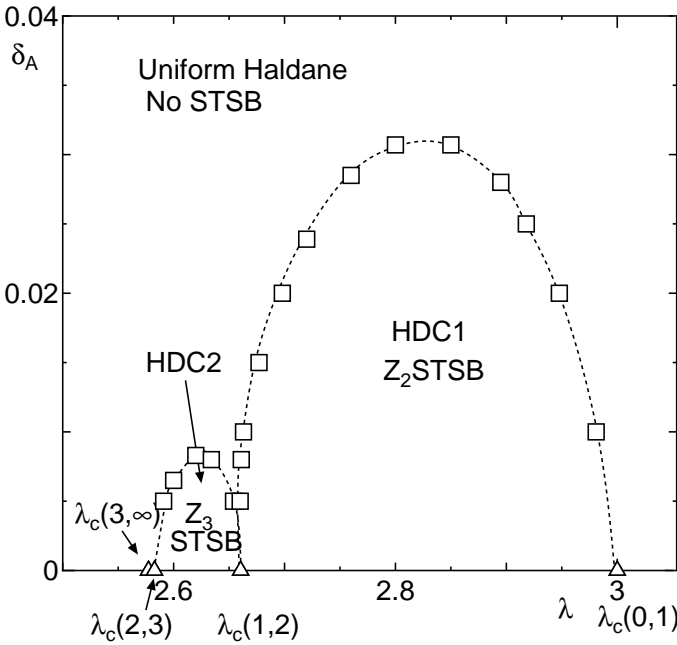

Fig. 8. Phase diagram of the MDC with type A distortion. The triangles indicate the position of the phase boundary for $\delta_{\mathrm{A}}=0$.

well as the UH phase are shown in Fig. 5. We see the translational invariance of period $n+1$ in the $\mathrm{HDC} n$ ground state in contrast to the period-1 invariance in the UH ground state. Hence, the $Z_{n+1}$ STSB takes place at the $\mathrm{HDC} n-\mathrm{UH}$ phase boundary. We expect that this transition belongs to the 2-dimensional $(n+1)$-clock model universality class. The system size dependence of $\Delta T$ for $\lambda=2.85$ is shown in Fig. 7(a) around the HDC1-UH phase boundary. Here, the data are plotted against $N^{-\beta / \nu}$ with the order parameter critical exponent $\beta=1 / 8$ and the correlation length critical exponent $\nu=1$ for the two-dimensional Ising universality class. This shows that the critical value of $\delta_{\mathrm{A}}$ lies between 0.0304 and 0.0308. A similar plot is shown in Fig. 7(b) for $\lambda=2.62$ around the HDC2-UH phase boundary, assuming the critical exponents of two-dimensional 3-clock model (equivalently 3 -state Potts model ${ }^{38)}$ ) with $\beta=1 / 9$ and $\nu=5 / 6$. This shows that the critical value of $\delta_{\mathrm{A}}$ lies between 0.00822 and 0.00826 . The critical points at other values of $\lambda$ are determined similarly. The results are shown in the phase diagram of Fig. 8. The error bars are within the size of the symbols.

To confirm the consistency of the universality class, the finite size scaling plot for the order parameter $\Delta T$ is carried out. According to the scaling hypothesis, the $\delta_{\mathrm{A}}$ dependence of the order parameter $\Delta T$ of the finite size systems near the critical point should obey the finite size scaling law ${ }^{39)}$

$$
\Delta T N^{\beta / \nu}=f\left(N\left(\delta_{\mathrm{A}}-\delta_{\mathrm{A}}^{\mathrm{c}}\right)^{\nu}\right),
$$

in terms of the scaled variables $\Delta T N^{\beta / \nu}$ and $N\left(\delta_{\mathrm{A}}-\delta_{\mathrm{A}}^{\mathrm{c}}\right)^{\nu}$ and the scaling function $f(x)$. In Figs. 9(a) and 9(b), $\Delta T N^{\beta / \nu}$ is plotted against $N\left(\delta_{\mathrm{A}}-\delta_{\mathrm{A}}^{\mathrm{c}}\right)^{\nu}$ around the HDC1-UH and HDC2-UH phase boundaries assuming the Ising and 3-clock universality classes, respectively. The critical points $\delta_{\mathrm{A}}^{\mathrm{c}}=0.0307$ (Fig. 9(a)) and 0.008248 (Fig. 9(b)) are chosen so that all data fall on a single universal scaling curve as well as possible. These plots are consistent with the expected universality class.

The critical behavior at the HDC1-UH transition in 

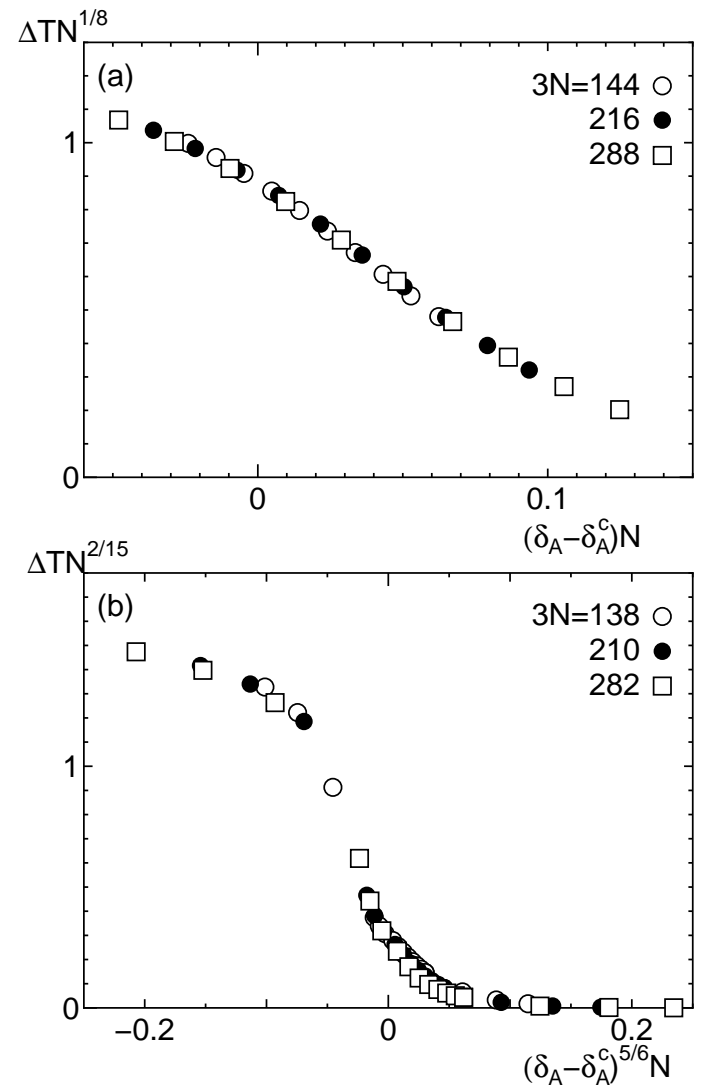

Fig. 9. Finite-size scaling plot of $\Delta T$ around the critical points. (a) Plot around the HDC1-UH phase boundary at $\lambda=2.85$. The Ising critical exponents $\nu=1$ and $\beta=1 / 8$ are assumed. The critical point is set at $\delta_{\mathrm{A}}^{\mathrm{c}}=0.0307$. (b) Plot around the HDC2-UH phase boundary at $\lambda=2.62$. The 3 -state Potts critical exponents $\nu=5 / 6$ and $\beta=1 / 9^{38}$ are assumed. The critical point is set at $\delta_{\mathrm{A}}^{\mathrm{c}}=0.008248$.

(a)

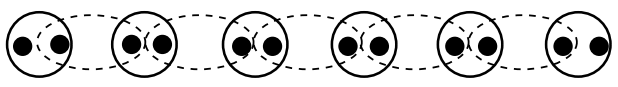

(b)

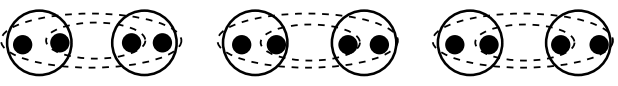

Fig. 10. Valence bond structures of the ground states of spin-1 bilinear-biquadratic chain in the (a) Haldane phase and (b) dimer phase. The spins with a magnitude of unity represented by open circles are decomposed into two spin-1/2 degrees of freedom represented by small filled circles. The valence bonds are represented by dashed ovals. The spins belonging to disconnected clusters in the dimer phase are connected by the valence bonds in the Haldane phase.

our model should be compared with that of the $S=$ 1 bilinear-biquadratic chain at the Takhtajan-Babujian point. ${ }^{40,41)}$ Both transitions are accompanied by $Z_{2^{-}}$ STSB which contributes to the conformal charge by $1 / 2$. For the HDC1-UH transition in our model, the rearrangement of valence bonds take place only within each diamond unit, as shown in Fig. 5(a) and 5(b). In contrast, in the $S=1$ bilinear-biquadratic chain, the spins belonging to disconnected clusters in the dimer phase are

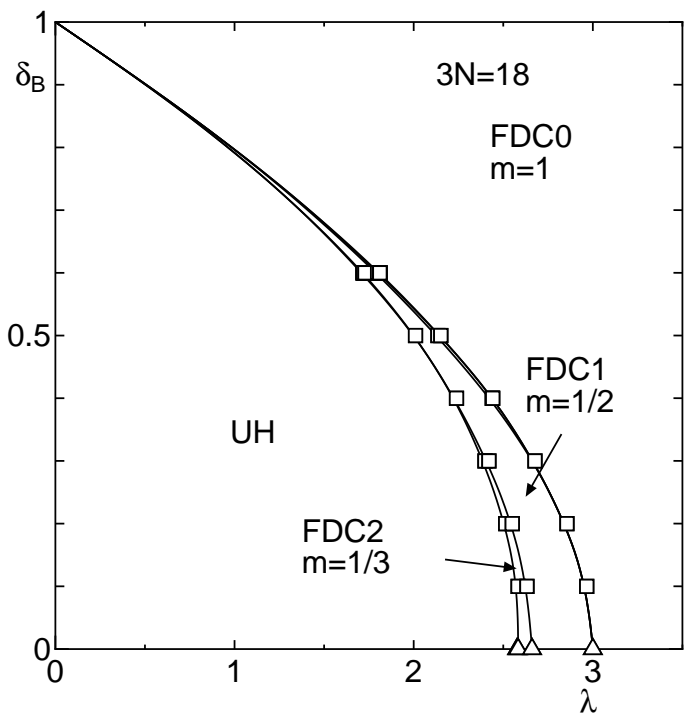

Fig. 11. Phase diagram of the MDC with type B distortion with $3 N=18$. The triangles indicate the position of the phase boundaries $\lambda_{\mathrm{C}}(n, n+1)$ for $\left.\delta_{\mathrm{B}}=0 .{ }^{12}\right)$

connected by the valence bonds in the Haldane phase, as shown in Fig. 10. Apart from $Z_{2}$-STSB, this is similar to the Gaussian criticality of the Haldane-dimer transition in the spin-1 alternating bond Heisenberg chain that contribute to the conformal charge by $1 .{ }^{42-44)}$ Therefore, the $S=1$ bilinear-biquadratic chain at the TakhtajanBabujian point is described by the conformal field theory with $c=1 / 2+1=3 / 2$, while the HDC1-UH transition in our model is described by the $c=1 / 2$ Ising conformal field theory.

\section{Ground-State Properties of the MDC with Type B Distortion}

In the case of type $\mathrm{B}$ distortion, the effective interaction between the spins of two cluster-n's separated by the dimer consisting of $\boldsymbol{\tau}_{l}^{(1)}$ and $\boldsymbol{\tau}_{l}^{(2)}$ is ferromagnetic, because both $\boldsymbol{S}_{l}$ and $\boldsymbol{S}_{l+1}$ tend to be antiparallel to $\boldsymbol{\tau}_{l}^{(1)}$. Therefore, we expect the ferrimagnetic ground state with spontaneous magnetization quantized as $m=1 /(n+1)$ per unit cell for small $\delta_{\mathrm{B}}$ in the range $\lambda_{\mathrm{c}}(n, n+1)<$ $\lambda<\lambda_{\mathrm{c}}(n-1, n)$. We call this phase a ferrimagnetic $\mathrm{DC} n$ phase (FDCn phase). In contrast, the ground state for $\lambda<\lambda_{c}(3, \infty)$ will remain in the Haldane phase, since a nonmagnetic gapped phase is generally robust against a weak distortion. For finite $\delta_{\mathrm{B}}$, we determined the groundstate phase diagram by the numerical diagonalization for the system size $3 N=18$, as shown in Fig. 11. Among system sizes tractable by numerical diagonalization, only this size of $3 N=18$ is compatible with all the groundstate structures with $n=0,1$, and 2. As expected, the FDC $n$ phases with $m=1 /(n+1)$ are found for these values of $n$.

By inspecting numerical data for the $3 N=18$ system, we also find other narrow ferrimagnetic phases between the $\operatorname{FDC} n$ and $\operatorname{FDC}(n+1)$ phases with $n=0,1$, and 2, although they are too narrow to be shown in Fig. 11. In order to investigate these phases in detail, we employ 

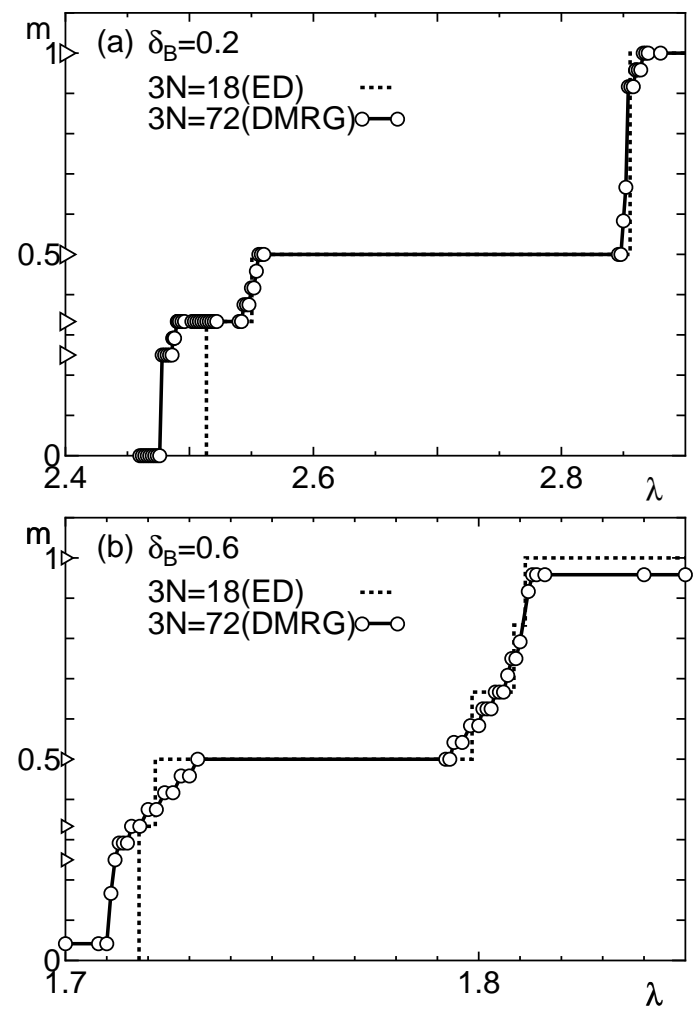

Fig. 12. Spontaneous magnetization for (a) $\delta_{\mathrm{B}}=0.2$ and (b) $\delta_{\mathrm{B}}=0.6$. The triangles on the vertical axes indicate the values of the spontaneous magnetization $m=1 /(n+1)$ in the FDC $n$ phases.

the DMRG calculation for $3 N=72$ keeping 120 states in each subsystem. Typical examples of the $\lambda$ dependence of spontaneous magnetization are shown in Fig. 12(a) for $\delta_{\mathrm{B}}=0.2$ and Fig. 12(b) for $\delta_{\mathrm{B}}=0.6$. Between the $\operatorname{FDC} n$ and $\operatorname{FDC}(n+1)$ phases with $n=0,1,2$, we find the partial ferrimagnetic phase in which the spontaneous magnetization varies continuously with $\lambda$. The ferrimagnetic phase of this kind has been found in various frustrated one-dimensional quantum spin systems. ${ }^{45-51)}$ In contrast, between the nonmagnetic phase and the FDC3 phase, we find no partial ferrimagnetic phase for small $\delta_{\mathrm{B}}$.

This can be understood as follows: At $\lambda=\lambda_{\mathrm{c}}(n, n+1)$, the cluster- $n$ and cluster- $(n+1)$ can coexist. As stated above, it is physically evident that the effective magnetic interaction between the clusters is ferromagnetic. Therefore, we can restrict the states of each cluster to the maximally polarized ground state with $\hat{S}_{i}^{z}=1$. Hence, the ground state of the whole chain is described by specifying the arrangement of cluster- $n$ 's and cluster- $(n+1)$ 's. We map the two possible values of the length of $i$-th cluster, $n_{i}=n$ and $n_{i}=n+1$, to two possible values of the spin- $1 / 2$ pseudospin, $\sigma_{i}^{z}=1 / 2$ and $\sigma_{i}^{z}=-1 / 2$, respectively. Then, the total magnetization $M$ is equal to the number of clusters $N_{\mathrm{c}}$. The total number of unit cells,
$N$, is related to the pseudospins $\sigma_{i}^{z}$ as

$$
N=\sum_{i=1}^{N_{\mathrm{c}}}\left(n+1+\frac{1}{2}-\sigma_{i}^{z}\right)=N_{\mathrm{c}}\left(n+\frac{3}{2}\right)-\sum_{i=1}^{N_{\mathrm{c}}} \sigma_{i}^{z} .
$$

Therefore, the ground-state magnetization per unit cell $m$ is given by

$$
m=\frac{N_{\mathrm{c}}}{\langle N\rangle}=\frac{1}{n+\frac{3}{2}-\sigma}
$$

with $\sigma \equiv \sum_{i=1}^{N_{\mathrm{c}}}\left\langle\sigma_{i}^{z}\right\rangle / N_{\mathrm{c}}$. The bracket $\langle\cdots\rangle$ represents the ground-state expectation value. In the presence of $\delta_{\mathrm{B}}$, the length of neighboring clusters can exchange through a second order process in $\delta_{\mathrm{B}}$. This corresponds to the spin exchange in terms of pseudospins. In this case, the interaction between the pseudospins is approximated by the spin-1/2 XXZ Hamiltonian

$$
\begin{gathered}
\mathcal{H}_{\mathrm{XXZ}}=\sum_{i=1}^{N_{\mathrm{c}}} \mathcal{H}_{\mathrm{XXZ}}(i, i+1), \\
\mathcal{H}_{\mathrm{XXZ}}(i, i+1)=J_{z}^{\mathrm{eff}} \sigma_{i}^{z} \sigma_{i+1}^{z}+J_{\perp}^{\mathrm{eff}}\left(\sigma_{i}^{x} \sigma_{i+1}^{x}+\sigma_{i}^{y} \sigma_{i+1}^{y}\right)
\end{gathered}
$$

up to the second order in $\delta_{\mathrm{B}}$. Here, further neighbor interactions are neglected. We estimate the effective exchange constants by comparing the energy spectrum of the pair Hamiltonian $\mathcal{H}_{\mathrm{XXZ}}(i, i+1)$ with that of the corresponding pair of clusters as follows:

(i) $\lambda=\lambda_{\mathrm{c}}(0,1)$

$$
\begin{aligned}
& J_{z}^{\mathrm{eff}} \simeq-0.039 \delta_{\mathrm{B}}{ }^{2} \\
& J_{\perp}^{\mathrm{eff}} \simeq 0.087 \delta_{\mathrm{B}}{ }^{2} .
\end{aligned}
$$

(ii) $\lambda=\lambda_{\mathrm{c}}(1,2)$

$$
\begin{aligned}
& J_{z}^{\mathrm{eff}} \simeq-0.0082 \delta_{\mathrm{B}}{ }^{2}, \\
& J_{\perp}^{\mathrm{eff}} \simeq 0.069 \delta_{\mathrm{B}}{ }^{2} .
\end{aligned}
$$

(iii) $\lambda=\lambda_{\mathrm{c}}(2,3)$

$$
\begin{aligned}
& J_{z}^{\mathrm{eff}} \simeq-0.0029 \delta_{\mathrm{B}}{ }^{2}, \\
& J_{\perp}^{\mathrm{eff}} \simeq 0.018 \delta_{\mathrm{B}}{ }^{2} .
\end{aligned}
$$

The details of the calculation are explained in Appendix.

In all cases $(\mathrm{i}) \sim$ (iii), we find that the effective coupling constants satisfy the inequality $-\left|J_{\perp}^{\text {eff }}\right|<J_{z}^{\text {eff }} \leq$ $\left|J_{\perp}^{\text {eff }}\right|$. As is well known, the ground state of the spin$1 / 2 \mathrm{XXZ}$ chain in this parameter regime is nonmagnetic and gapless in the absence of a magnetic field. Roughly speaking, $\Delta \lambda \equiv \lambda-\lambda_{\mathrm{c}}(n, n+1)$ corresponds to the effective magnetic field $h_{\text {eff }}$ conjugate to the total pseudospin $\sum_{i} \sigma_{i}^{z}$, because the increase in $\lambda$ favors cluster- $n$ over cluster- $(n+1)$; however, this correspondence should not be taken literally. A more precise argument is also given in Appendix. When $\Delta \lambda$ takes a large negative value, the pseudospins are fully polarized downward to give $\left\langle\sigma_{l}^{z}\right\rangle=-1 / 2$. This state corresponds to the $\operatorname{FDC}(n+1)$ state with $m=1 /(n+2)$. When $h_{\text {eff }}$ reaches the critical value $h_{\mathrm{c} 1} \equiv-\left(\left|J_{\perp}^{\mathrm{eff}}\right|+J_{z}^{\mathrm{eff}}\right)$, the magnetization starts to increase continuously until all spins are fully polarized 
upward at the critical effective field $h_{\mathrm{c} 2} \equiv\left|J_{\perp}^{\mathrm{eff}}\right|+J_{z}^{\text {eff }}$. This corresponds to the FDC $n$ state with $m=1 /(n+1)$.

On the other hand, the magnetization jumps from 0 to $1 / 4$ at the phase boundary between the Haldane phase and the FDC3 phase for small $\delta_{\mathrm{B}}$. At this phase boundary, no finite size clusters coexist with cluster-3. Therefore, no pseudospin degrees of freedom can be defined. Consequently, no partial ferrimagnetic phase can be realized. In contrast, for larger values of $\delta_{\mathrm{B}}$, we numerically find a partial ferrimagnetic phase between the FDC3 and UH phases. This would be ascribed to the contribution of other finite-length clusters with low lying energies which come into play through higher-order processes in $\delta_{\mathrm{B}}$.

\section{Summary and Discussion}

We introduced two types of distortion, type A and type $\mathrm{B}$, into the $\mathrm{MDC}$ with spins 1 and $1 / 2$, and investigated the ground-state phases. The phase diagrams are characteristic of the type A and type B distortions, respectively. For the type A distortion, the effective interaction between the cluster spins is antiferromagnetic with bilinear and biquadratic terms. The numerically estimated values of the effective couplings show that the DCn ground states are transformed into the HDC $n$ ground states. The order parameters characterizing the $\mathrm{HDC} n$ phases are defined and the UH-HDCn phase boundaries are determined using the DMRG data. From the valence bond structure of each phase, we expect that the UH-HDCn phase transition belongs to the universality class of the 2-dimensional $(n+1)$-clock model. The finite size scaling plot of the order parameter is consistent with this identification. For the type B distortion, the effective interaction between the cluster spins is ferromagnetic. In addition to the FDCn phases with quantized spontaneous magnetization $m=1 /(n+1)$, the partial ferrimagnetic phases are also found numerically between the FDC $n$ and $\mathrm{FDC}(n+1)$ phases. A physical interpretation of the partial ferrimagnetic phase is given for small $\delta_{\mathrm{B}}$ by mapping onto an effective pseudospin-1/2 XXZ chain.

Generally, the introduction of lattice distortion into a physical model increases the possibility that a corresponding material is realized. In the MDC, there are three types of distortion modes affecting the exchange interactions. Among them, the two types investigated in the present paper are of generic nature, because the local conservation laws that hold in the undistorted MDC are broken. This suggests that the observation of the exotic phenomena predicted in the present paper is possible even if the corresponding material is not exactly described by the model Hamiltonians (1) and (2).

If a distorted MDC material is synthesized, the distortion may be controlled by, e.g., applying pressure. If the distortion is of type $\mathrm{A}$, the Curie constant vanishes as the DCn ground state turns into one of the HDCn ground states. The magnetic susceptibility and magnetic specific heat will have an activation-type temperature dependence with activation energy proportional to the effective coupling between the cluster spins, which is of the order of $\delta_{\mathrm{A}}{ }^{2}$. These HDCn phases are not realized if the distortion $\delta_{\mathrm{A}}$ exceeds $\sim 0.03$ even in the most robust case of $n=1$. In a real material, the STSB in the valence bond structure manifests itself as a magnetic superstructure. It is also possible that it is accompanied by a lattice superstructure of corresponding periodicity if the spin-lattice coupling is present. Therefore careful measurements of magnetic and lattice superstructures would help with the observation of $\mathrm{HDC} n$ phases with $1 \leq n \leq 3$

On the other hand, if the distortion of the material is of type $B$, the ground sate is ferrimagnetic. At low but finite temperatures, however, the spontaneous magnetization vanishes owing to the one-dimensionality. As a precursor of ordering at $T=0$, the low-temperature magnetic susceptibility should diverge as $T^{-2}$ with a coefficient proportional to the effective coupling $\sim \delta_{\mathrm{B}}{ }^{2}$ between the cluster spins. ${ }^{52-54)}$ This means that even a weak magnetic field of the order of $H \sim T^{2} / \delta_{\mathrm{B}}{ }^{2}$ derives the finite-temperature magnetization up to the value of the ground-state spontaneous magnetization. This enables the experimental estimation of the spontaneous magnetization in real materials. The quantized ferrimagnetic behavior should be observed for wide ranges of the parameters $\lambda$ and $\delta_{\mathrm{B}}$ as shown in Fig. 11, and should be easily observed if an appropriate material is synthesized. The partial ferrimagnetic phases are limited to narrow intervals of the parameters $\delta_{\mathrm{B}}$ and $\lambda$. Therefore, these can only be observed as a temperature-independent crossover between two quantized ferrimagnetic behaviors with careful exclusion of the thermal effect.

We have demonstrated that various exotic ground states and phase transitions between them are realized in the distorted MDC with spins 1 and 1/2, which has a strong frustration. The physical pictures of these phenomena have become clear. This is made possible because the ground state of the undistorted MDC is known exactly. Therefore, we expect that our model may provide a means of understanding the similar exotic phenomena realized owing to the interplay of spin ordering, quantum fluctuation, and strong frustration in more general frustrated quantum chains on a firm ground. For example, partial ferrimagnetic phases are found in various onedimensional frustrated quantum spin models. ${ }^{45-51)}$ However, some of them are only numerically confirmed and no physical explanation has been given so far. We hope that the present study paves the way to the general understanding of these partial ferrimagnetic states.

We thank J. Richter for drawing our attention to ref. 28 and related works. The numerical diagonalization program is based on the package TITPACK ver.2 coded by H. Nishimori. The numerical computation in this work has been carried out using the facilities of the Supercomputer Center, Institute for Solid State Physics, University of Tokyo and Supercomputing Division, Information Technology Center, University of Tokyo. KH is supported by a Grant-in-Aid for Scientific Research on Priority Areas, "Novel States of Matter Induced by Frustration" (20048003) from the Ministry of Education, Culture, Sports, Science and Technology of Japan and a Grant-in-Aid for Scientific Research (C) (21540379) from the Japan Society for the Promotion of Science. KT and HS are supported by a Fund for Project Research from Toyota Technological Institute. 


\section{Appendix}

The Hamiltonian $\mathcal{H}_{\mathrm{B}}$ with the type $\mathrm{B}$ distortion is rewritten as

$$
\mathcal{H}_{\mathrm{B}}=\mathcal{H}_{0}+\delta \mathcal{H},
$$

where

$$
\delta \mathcal{H}=\delta_{\mathrm{B}} \sum_{l=1}^{N}\left(\boldsymbol{S}_{l}+\boldsymbol{S}_{l+1}\right)\left(\boldsymbol{\tau}_{l}^{(1)}-\boldsymbol{\tau}_{l}^{(2)}\right) .
$$

For small $\delta_{\mathrm{B}}$, the ground state around $\lambda=\lambda_{\mathrm{c}}(n, n+1)$ consists almost entirely of cluster- $n$ 's and cluster- $(n+$ 1)'s. Hence, as a good approximation, we consider $\mathcal{H}_{\mathrm{B}}$ only in the restricted Hilbert space where each state involves no clusters except for cluster-n's and cluster$(n+1)$ 's. Under the fixed cluster number $N_{\mathrm{c}}$ in this Hilbert space, $\mathcal{H}_{0}$ is equivalent to the following effective Hamiltonian expressed in terms of pseudospin operators:

$$
\begin{aligned}
\mathcal{H}_{\mathrm{eff}}^{0} & =E_{\mathrm{G}}^{0}(n+1 ; \lambda) \sum_{i=1}^{N_{\mathrm{c}}}\left(\frac{1}{2}-\sigma_{i}^{z}\right) \\
& +E_{\mathrm{G}}^{0}(n ; \lambda) \sum_{i=1}^{N_{\mathrm{c}}}\left(\frac{1}{2}+\sigma_{i}^{z}\right),
\end{aligned}
$$

where $\sigma_{i}^{z}=1 / 2$ and $\sigma_{i}^{z}=-1 / 2$ correspond to $n_{i}=n$ and $n_{i}=n+1$, respectively. $E_{\mathrm{G}}^{0}(n ; \lambda)$ is the groundstate energy of a cluster- $n$ and a dimer in the absence of distortion, and is given by

$$
E_{\mathrm{G}}^{0}(n ; \lambda)=\tilde{E}(2 n+1)+\frac{\lambda n}{4}-\frac{3 \lambda}{4},
$$

where $\tilde{E}(2 n+1)$ is the ground-state energy of the spin-1 antiferromagnetic Heisenberg chain with length $2 n+1 .{ }^{12}$ )

The application of $\delta \mathcal{H}$ to the unperturbed ground state transforms one of the $T_{l}=0$ bonds to a $T_{l}=1$ bond or vice versa. Then the resulting states contain clusters with lengths less than $n$ or greater than $2 n$. Since these states are outside the restricted Hilbert space, no correction to the ground-state energy is present within the first order in $\delta_{\mathrm{B}}$. Hence, the lowest-order correction is of the order of $\delta_{\mathrm{B}}{ }^{2}$. Up to the second order in $\delta_{\mathrm{B}}$, the effective pseudospin Hamiltonian is given by

$$
\begin{aligned}
\mathcal{H}_{\mathrm{eff}} & =E_{\mathrm{G}}\left(n+1 ; \lambda, \delta_{\mathrm{B}}\right) \sum_{i=1}^{N_{\mathrm{c}}}\left(\frac{1}{2}-\sigma_{i}^{z}\right) \\
& +E_{\mathrm{G}}\left(n ; \lambda, \delta_{\mathrm{B}}\right) \sum_{i=1}^{N_{\mathrm{c}}}\left(\frac{1}{2}+\sigma_{i}^{z}\right)+\mathcal{H}_{\mathrm{XXZ}}
\end{aligned}
$$

where $E_{\mathrm{G}}\left(n ; \lambda, \delta_{\mathrm{B}}\right)$ is the ground-state energy of a cluster- $n$ and a dimer including the second order correction in $\delta_{\mathrm{B}}$. This is simply expressed as

$$
\mathcal{H}_{\mathrm{eff}}=N_{\mathrm{c}} \bar{E}_{\mathrm{G}}+\Delta E_{\mathrm{G}} \sum_{i=1}^{N_{\mathrm{c}}} \sigma_{i}^{z}+\mathcal{H}_{\mathrm{XxZ}},
$$

with

$$
\begin{aligned}
\bar{E}_{\mathrm{G}} & =\frac{1}{2}\left(E_{G}\left(n+1 ; \lambda, \delta_{\mathrm{B}}\right)+E_{G}\left(n ; \lambda, \delta_{\mathrm{B}}\right)\right), \\
\Delta E_{\mathrm{G}} & =E_{G}\left(n ; \lambda, \delta_{\mathrm{B}}\right)-E_{G}\left(n+1 ; \lambda, \delta_{\mathrm{B}}\right) .
\end{aligned}
$$

The effective coupling constants $J_{z}^{\text {eff }}$ and $J_{\perp}^{\text {eff }}$ in $\mathcal{H}_{\mathrm{XxZ}}$ are also of the second order in $\delta_{\mathrm{B}}$. We determine $J_{z}^{\text {eff }}$ and $J_{\perp}^{\text {eff }}$ so as to reproduce the low-lying energy spectrum of a pair of cluster- $n$ 's by that of two-pseudospin Hamiltonian

$\mathcal{H}_{\mathrm{eff}}(i, i+1)=2 \bar{E}_{\mathrm{G}}+\Delta E_{\mathrm{G}}\left(\sigma_{i}^{z}+\sigma_{i+1}^{z}\right)+\mathcal{H}_{\mathrm{XXZ}}(i, i+1)$

In each of the subspaces $\sigma_{i}^{z}+\sigma_{i+1}^{z}= \pm 1, \sigma_{i}^{z}=$ $\sigma_{i+1}^{z}=\sigma= \pm 1 / 2$. Therefore, the Hilbert space is onedimensional and the eigenvalue of $\mathcal{H}_{\mathrm{eff}}(i, i+1)$ is simply $E_{\sigma \sigma}=2 \bar{E}_{\mathrm{G}}+2 \Delta E_{\mathrm{G}} \sigma+J_{z}^{\text {eff }} / 4$ with $\sigma= \pm 1 / 2$. In the subspace $\sigma_{i}^{z}+\sigma_{i+1}^{z}=0$, the Hilbert space is two-dimensional and the eigenvalues of $\mathcal{H}_{\mathrm{eff}}(i, i+1)$ are $E_{ \pm}=2 \bar{E}_{\mathrm{G}}-J_{z}^{\mathrm{eff}} / 4 \pm J_{\perp}^{\mathrm{eff}} / 2$.

The original Hamiltonian of the cluster consisting of a cluster- $n$ and a cluster- $n^{\prime}$ is the distorted diamond chain with length $n+n^{\prime}$.

$$
\begin{gathered}
\mathcal{H}\left(n+n^{\prime}\right)=\sum_{l=1}^{n+n^{\prime}+1}\left[\left(1+\delta_{\mathrm{B}}\right) \boldsymbol{S}_{l} \boldsymbol{\tau}_{l}^{(1)}+\left(1+\delta_{\mathrm{B}}\right) \boldsymbol{\tau}_{l}^{(1)} \boldsymbol{S}_{l+1}\right. \\
\left.+\left(1-\delta_{\mathrm{B}}\right) \boldsymbol{S}_{l} \boldsymbol{\tau}_{l}^{(2)}+\left(1-\delta_{\mathrm{B}}\right) \boldsymbol{\tau}_{l}^{(2)} \boldsymbol{S}_{l+1}+\lambda \boldsymbol{\tau}_{l}^{(1)} \boldsymbol{\tau}_{l}^{(2)}\right]
\end{gathered}
$$

We denote the $\alpha$-th eigenvalue of $\mathcal{H}\left(n+n^{\prime}\right)$ as $E(n+$ $\left.n^{\prime} ; \alpha\right)$. Comparing the corresponding expression for the eigenvalues, we find

$$
\begin{gathered}
E(2 n ; 0)=E_{\frac{1}{2}, \frac{1}{2}}=2 \bar{E}_{\mathrm{G}}+\Delta E_{\mathrm{G}}+\frac{J_{z}^{\mathrm{eff}}}{4} \\
E(2 n+2 ; 0)=E_{-\frac{1}{2},-\frac{1}{2}}=2 \bar{E}_{\mathrm{G}}-\Delta E_{\mathrm{G}}+\frac{J_{z}^{\mathrm{eff}}}{4} \\
E(2 n+1 ; 0)=E_{-}=2 \bar{E}_{\mathrm{G}}-\frac{J_{z}^{\mathrm{eff}}}{4}-\frac{J_{\perp}^{\mathrm{eff}}}{2} \\
E(2 n+1 ; 1)=E_{+}=2 \bar{E}_{\mathrm{G}}-\frac{J_{z}^{\mathrm{eff}}}{4}+\frac{J_{\perp}^{\mathrm{eff}}}{2} .
\end{gathered}
$$

Solving these sets of equations, with respect to $J_{z}^{\text {eff }}$ and $J_{\perp}^{\text {eff }}$, we find

$$
\begin{aligned}
J_{\perp}^{\mathrm{eff}}= & E(2 n+1 ; 1)-E(2 n+1 ; 0), \\
J_{z}^{\mathrm{eff}}=2[ & E(2 n+2 ; 0)+E(2 n ; 0) \\
& \quad-E(2 n+1 ; 1)-E(2 n+1 ; 0)] .
\end{aligned}
$$

Note that the rhs's of (A.15) and (A.16) vanish for $\delta_{\mathrm{B}}=0$. We numerically evaluated $E(2 n ; 0), E(2 n+1 ; 0)$, $E(2 n+1 ; 1)$, and $E(2 n+2 ; 0)$ at $\lambda=\lambda_{\mathrm{c}}(n, n+1)(n=$ $0,1,2)$ for small $\delta_{\mathrm{B}}$. Using these values in eqs. (A.15) and (A.16), we determined $J_{\perp}^{\text {eff }}$ and $J_{z}^{\text {eff }}$ as eqs. (13)-(15).

For the whole MDC, the ground-state energy is written as

$$
E_{0}=N_{\mathrm{c}} \bar{E}_{\mathrm{G}}+N_{\mathrm{c}} \Delta E_{G} \sigma+N_{\mathrm{c}} \epsilon_{\mathrm{XXZ}}(\sigma)
$$

where $\epsilon_{\mathrm{XXZ}}(\sigma)$ is the ground-state energy per site of a magnetized spin-1/2 XXZ chain with $\left\langle\sigma_{i}^{z}\right\rangle=\sigma$. The number of unit cells, $N$, of the original MDC is given by the expectation value of eq. (9) as $N=N_{\mathrm{c}}\left(n+\frac{3}{2}-\sigma\right)$. Therefore, we have

$$
E_{0}=\frac{N}{n+\frac{3}{2}-\sigma}\left(\bar{E}_{\mathrm{G}}+\Delta E_{G} \sigma+\epsilon_{\mathrm{XXZ}}(\sigma)\right)
$$




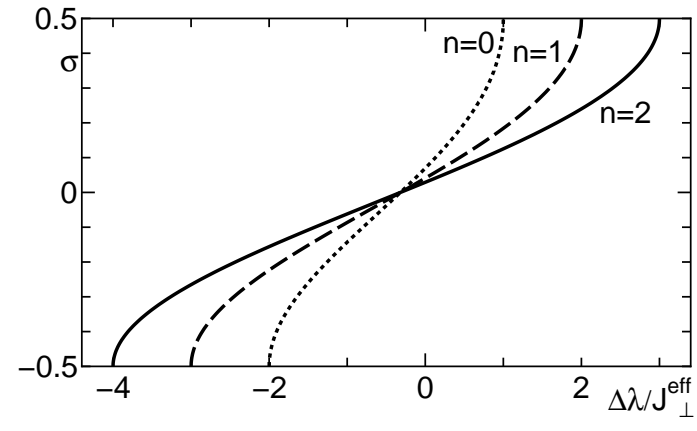

Fig. A.1. Relationship between $\sigma$ and $\Delta \lambda$ for $n=0,1$, and 2 .

Minimizing this with respect to $\sigma$ with fixed $N$, we find

$$
\Delta \lambda=\left(n+\frac{3}{2}-\sigma\right) \frac{\partial \epsilon_{\mathrm{XxZ}}(\sigma)}{\partial \sigma}+\epsilon_{\mathrm{XXZ}}(\sigma)
$$

where $\Delta \lambda=\lambda-\lambda_{\mathrm{c}}\left(n, n+1 ; \delta_{\mathrm{B}}\right)$ and $\lambda_{\mathrm{c}}\left(n, n+1 ; \delta_{\mathrm{B}}\right)$ is defined by

$$
(n+2) E_{G}\left(n ; \lambda_{\mathrm{c}}, \delta_{\mathrm{B}}\right)-(n+1) E_{G}\left(n+1 ; \lambda_{\mathrm{c}}, \delta_{\mathrm{B}}\right)=0 .
$$

To simplify the calculation, we replace $\epsilon_{\mathrm{XxZ}}(\sigma)$ by the ground-state energy of the spin- $1 / 2 \mathrm{XY}$ chain $\epsilon_{\mathrm{XY}}=$ $-\left(J_{\perp}^{\text {eff }} / \pi\right) \cos \pi \sigma$, because $\left|J_{\perp}^{\text {eff }}\right|$ is substantially larger than $\left|J_{z}^{\text {eff }}\right|$ in all cases. Then we find

$$
\frac{\Delta \lambda}{J_{\perp}^{\mathrm{eff}}}=\left(n+\frac{3}{2}-\sigma\right) \sin \pi \sigma-\frac{1}{\pi} \cos \pi \sigma .
$$

This relation is plotted in Fig. A.1 for $n=0,1$ and 2. It is clear that $\sigma$ continuously increases from $-1 / 2$ to $1 / 2$ with an increase in $\lambda$.

1) Frustrated Spin Systems, ,ed. H. T. Diep: (World Scientific, Singapore, 2005), Chaps. 5 and 6.

2) Proc. Int. Conf. on Highly Frustrated Magnetism (HFM2008) J. Phys.: Conf. Series 145 (2009).

3) C. K. Majumdar and D. K. Ghosh: J. Math. Phys. 10 (1969) 1399.

4) For examples of experimental materials, see M. Hase, H. Kuroe, K. Ozawa, O. Suzuki, H. Kitazawa, G. Kido, and T. Sekine: Phys. Rev. B 70 (2004) 104426.

5) B. S. Shastry and B. Sutherland: Physica B+C 108 (1981) 1069.

6) H. Kageyama, K. Yoshimura, R. Stern, N.V. Mushnikov, K. Onizuka, M. Kato, K. Kosuge, C.P. Slichter, T. Goto, and Y. Ueda: Phys. Rev. Lett. 82 (1999) 3168.

7) H. Kageyama, M. Nishi, N. Aso, K. Onizuka, T. Yosihama, K. Nukui, K. Kodama, K. Kakurai, and Y. Ueda: Phys. Rev. Lett. 84 (2000) 5876.

8) K. Takano: J. Phys. A: Math. Gen. 27 (1994) L269.

9) K. Takano, K. Kubo, and H. Sakamoto: J. Phys.: Condens. Matter 8 (1996) 6405.

10) H. Niggemann, G. Uimin, and J. Zittartz: J. Phys.: Condens. Matter 9 (1997) 9031.

11) H. Niggemann, G. Uimin, and J. Zittartz: J. Phys.: Condens. Matter 10 (1998) 5217.

12) K. Takano, H. Suzuki, and K. Hida: Phys. Rev. B 80 (2009) 104410.

13) K. Hida, K. Takano, and H. Suzuki: J. Phys. Soc. Jpn. 78 (2009)
084716

14) K. Hida, K. Takano, and H. Suzuki: J. Phys. Soc. Jpn. 79 (2010) 044702.

15) K. Okamoto, T. Tonegawa, Y. Takahashi, and M. Kaburagi: J. Phys.: Condens. Matter 11 (1999) 10485.

16) K. Okamoto, T. Tonegawa, and M. Kaburagi: J. Phys.: Condens. Matter 15 (2003) 5979

17) K. Sano and K. Takano: J. Phys. Soc. Jpn. 69 (2000) 2710.

18) H. Kikuchi, Y. Fujii, M. Chiba, S. Mitsudo, T. Idehara, T. Tonegawa, K. Okamoto, T. Sakai, T. Kuwai, and H. Ohta: Phys. Rev. Lett. 94 (2005) 227201.

19) H. Ohta, S. Okubo, T. Kamikawa, T. Kunimoto, Y. Inagaki, H. Kikuchi, T. Saito, M. Azuma, and M. Takano: J. Phys. Soc. Jpn. 72 (2003) 2464.

20) A. Izuoka, M. Fukada, R. Kumai, M. Itakura, S. Hikami, and T. Sugawara: J. Am. Chem. Soc. 116 (1994) 2609.

21) D. Uematsu and M. Sato: J. Phys. Soc. Jpn. 76 (2007) 084712.

$22)$ N. B. Ivanov, J. Richter, and J. Schulenburg: Phys. Rev. B 79 (2009) 104412.

23) N.B. Ivanov and J. Richter: Phys. Lett. A 232 (1997) 308.

24) J. Richter, N. B. Ivanov, and J. Schulenburg: J. Phys.: Condens. Matter 10 (1998) 3635.

25) A. Koga, K. Okunishi, and N. Kawakami: Phys. Rev. B 62 (2000) 5558.

26) A. Koga and N. Kawakami: Phys. Rev. B 65 (2002) 214415.

27) J. Schulenburg and J. Richter: Phys. Rev. B 65 (2002) 054420.

28) J. Schulenburg and J. Richter: Phys. Rev. B 66 (2002) 134419.

29) T. Hakobyan, J. H. Hetherington, and M. Roger: Phys. Rev. B 63 (2001) 144433.

30) L. C̆anovà, J. Strec̆ka, and M. Jasčŭr: J. Phys.: Condens. Matter 18 (2006) 4967.

31) L. Canovà, J. Strečka, and T. Lučivjanský: Condens. Matter Phys. 12 (2009) 353.

32) H. Kobayashi, Y. Fukumoto, and A. Oguchi: J. Phys. Soc. Jpn. 78 (2009) 074004.

33) C. Mathonière, J.-P. Sutter, and J. V. Yakhmi: in Magnetism: Molecules to Materials IV, ed. J. S. Miller and M. Drillon (Wiley, Weinheim, 2003) p. 1.

34) Y. Hosokoshi and K. Inoue: in Carbon Based Magnetism, ed. T. L. Makarova and F. Palacio (Elsevier B. V., Amsterdam, 2006) p. 107.

35) E. Lieb and D. Mattis: J. Math. Phys. 3, (1962) 749.

36) T. Kennedy: J. Phys.: Condens. Matter 2 (1990) 5737.

37) G. Fáth and J. Sólyom: Phys. Rev. B 44 (1991) 11836.

38) F. Y. Wu: Rev. Mod. Phys. 54 (1982) 235.

39) M. N. Barber: Phase Transitions and Critical Phenomena 8, ed. C. Domb and J. L. Lebowitz (Academic Press, London, 1983) p. 146.

40) L.A. Takhtajan: Phys. Lett. 87A (1982) 479.

41) H. M. Babujian: Phys. Lett. 90A (1982) 479.

42) I. Affleck and F. D. M. Haldane: Phys. Rev. B36 (1987) 5291.

43) Y. Kato and A. Tanaka: J. Phys. Soc. Jpn 66 (1997) 3944.

44) A. Kitazawa and K. Nomura: J. Phys. Soc. Jpn. 66 (1997) 3944.

45) S. Sachdev and T. Senthil: Ann. Phys. 251 (1996) 76.

46) L. Bartosch, M. Kollar, and P. Kopietz: Phys. Rev. B 67 (2003) 092403.

47) N. B. Ivanov and J. Richter: Phys. Rev. B 69 (2004) 214420.

48) S. Yoshikawa and S. Miyashita: J. Phys. Soc. Jpn. Suppl. 74 (2005) 71.

49) K. Hida: J. Phys. Condens. Matter: 19 (2007) 145225.

50) K. Hida and K. Takano: Phys. Rev. B 78 (2008) 064407.

51) R. R. Montenegro-Filho and M. D. Coutinho-Filho: Phys. Rev. B 78 (2008) 014418.

$52)$ M. Takahashi: Prog. Theor. Phys. Suppl. 87 (1986) 233.

53) S. Yamamoto: Phys. Rev. B 59 (1999) 1024.

54) S. Yamamoto and T. Fukui: Phys. Rev. B 57 (1998) R14008. 